Canadian University Music Review

Revue de musique des universités canadiennes

\title{
Théorie des intervalles
}

\section{Gino Stefani}

Numéro 7, 1986

URI : https://id.erudit.org/iderudit/1014088ar

DOI : https://doi.org/10.7202/1014088ar

Aller au sommaire du numéro

\section{Éditeur(s)}

Canadian University Music Society / Société de musique des universités

canadiennes

\section{ISSN}

0710-0353 (imprimé)

2291-2436 (numérique)

Découvrir la revue

\section{Citer cet article}

Stefani, G. (1986). Théorie des intervalles. Canadian University Music Review / Revue de musique des universités canadiennes, (7), 80-102.

https://doi.org/10.7202/1014088ar

All Rights Reserved (C Canadian University Music Society / Société de musique des universités canadiennes, 1986
Ce document est protégé par la loi sur le droit d'auteur. L’utilisation des services d'Érudit (y compris la reproduction) est assujettie à sa politique d'utilisation que vous pouvez consulter en ligne.

https://apropos.erudit.org/fr/usagers/politique-dutilisation/ 


\title{
THÉORIE DES INTERVALLES
}

\author{
Gino Stefani
}

\section{Prémisses}

Notre point de départ est une donnée de l'expérience quotidienne : les intervalles musicaux sont des éléments signifiants.

Un exemple : la phrase musicale "All'erta!", au début de Trovatore. Tout auditeur, même ignorant le libretto, ressent cette phrase comme un geste oral ample, emphatique, imposant, décidé, péremptoire ; finalement, comme un cri, un ordre, un rappel autoritaire. Or, il suffit d'un instant de réflexion pour se rendre compte que l'intervalle d'octave concourt d'une façon évidente et d'une manière cohérente à bâtir ce sens. Pour vérifier cela, il n'y a qu'à remplacer l'octave par d'autres intervalles, en remarquant ce qui change dans le sens d'ensemble de la phrase ou, plus précisément, de la situation en scène ; on en conclura sans effort que cet intervalle apparaît justement comme le plus adapté à la situation.

Donc, l'intervalle d'octave a un sens à lui, connu par Verdi et par nous, antérieur à son emploi dans cet opéra ou un autre, chez cet auteur ou un autre ; un sens plus ou moins homologable et composable avec le sens de certains contextes et certaines circonstances extramusicales verbales et théâtrales, par exemple. Jusqu'ici, l'expérience est commune.

Mais voici le problème qui se pose aux musicologues : quel est, précisément, ce sens ? comment va-t-il se construire ? est-il toujours présent de la même manière dans les différentes occurrences de l'intervalle ? 
A ces questions, la musicologie traditionnelle n'a donné jusqu'ici que des réponses partielles ; nous renvoyons à plus tard une critique quelque peu détaillée de ces réponses, mais on peut dire d'ores et déjà que l'acoustique ne saisit pas la dimension linguistique et culturelle des intervalles, la théorie musicale en traite le fonctionnement "syntaxique" en négligeant le "sémantique", et, à l'inverse, certains traités sur la sémantique des intervalles (tel celui de Cooke) en négligent l'aspect syntaxique, d'où des résultats qui ne sont pas convaincants.

Or, l'hypothèse suggérée par l'expérience commune (confirmée $a$ contrario par ces impasses ou partialités théoriques) est justement que l'intervalle soit une unité signifiante de type linguistique, dans laquelle on trouve une certaine conformité entre le plan de l'expression (ou "syntaxique") et celui du contenu (ou "sémantique"). Le but de ces pages sera précisément de développer cette hypothèse dans un modèle théorique articulé.

\section{Le modèle}

A. En tant qu'élément de cette structure de "langage" qu'est une échelle musicale, un intervalle se présente sous deux aspects : (i) une relation qualitative ou fonctionnelle entre deux notes de l'échelle, et (ii) une grandeur diastématique, c'est-à-dire une distance, un ambitus, un espace (virtuel, scalaire) entre les deux notes.

a) En ce qui concerne le premier aspect, l'octave, c'est d'abord la relation d'identité entre les deux notes extrêmes du modèle (pattern) scalaire complet, en cycles ou récurrences divers (en première instance, deux cycles contigus) ; c'est là ce qu'on exprime couramment en disant que l'octave est "un même son à des hauteurs différentes". Quant au deuxième aspect, l'octave, c'est la totalité du cycle (pattern) scalaire luimême (do-ré-mi- ... - -do). 
Or, ce sens-signifié ("syntaxique") de l'intervalle, sitôt qu'on le pense et ressent en codes logico-sensoriels-émotifs ${ }^{1}$ - ce qui arrive inévitablement -, subit une diffraction aboutissant à un spectre ("sémantique") où l'on trouve des catégories telles que : simplicité, répétition, redondance, renforcement, redoublement, stabilité, affirmation, conclusivité, espace maximum, amplification, décision, euphorie, etc.

Ce fonctionnement syntaxico-sémantique est "profond" et "abstrait", tel que peut l'être celui d'une racine lexicale, ou une catégorie grammaticale, ou un paradigme compositionnel hors-temps : c'est justement le niveau de la théorie musicale. Il est évident - et on ne devra pas l'oublier - que ce noyau profond est présent dans toutes les manifestations dites de surface, c'est-à-dire dans toutes les occurrences concrètes de l'intervalle dans les répertoires musicaux.

b) Sur ce noyau "logique" s'insèrent les articulations: l'articulation temporelle simultané/successif, et celle, cinético-spatiale, ascendant/descendant, articulations communes à tous les intervalles et fonctionnant à leur tour comme des matrices de sens.

En conjugant ce niveau de sens avec celui qui précède, on engendre de nouveaux traits sémantiques tels que : fusion, consonance, déplacement maximum, grand bond, grand élan, retour au point de départ, etc. ; ces traits sont à attribuer respectivement à l'octave harmonique ou mélodique.

c) Noyau et articulations forment un niveau commun, puisque toute occurrence concrète de l'octave implique le noyau et l'une ou l'autre des articulations. Nous appellerons "grammatical" ce niveau ; mais pour

1 Ici et par après, nous ferons référence à la théorie qui met en forme la production de sens par la musique dans un modèle qui fontionne à différents niveaux : codes généraux, pratiques sociales, techniques musicales, oeuvre. Cf. Stefani (1981 et $1982:$ 49-84). 
l'instant, il nous suffit de l'appeler "niveau premier". Voici, en schéma, la représentation de ce premier niveau :

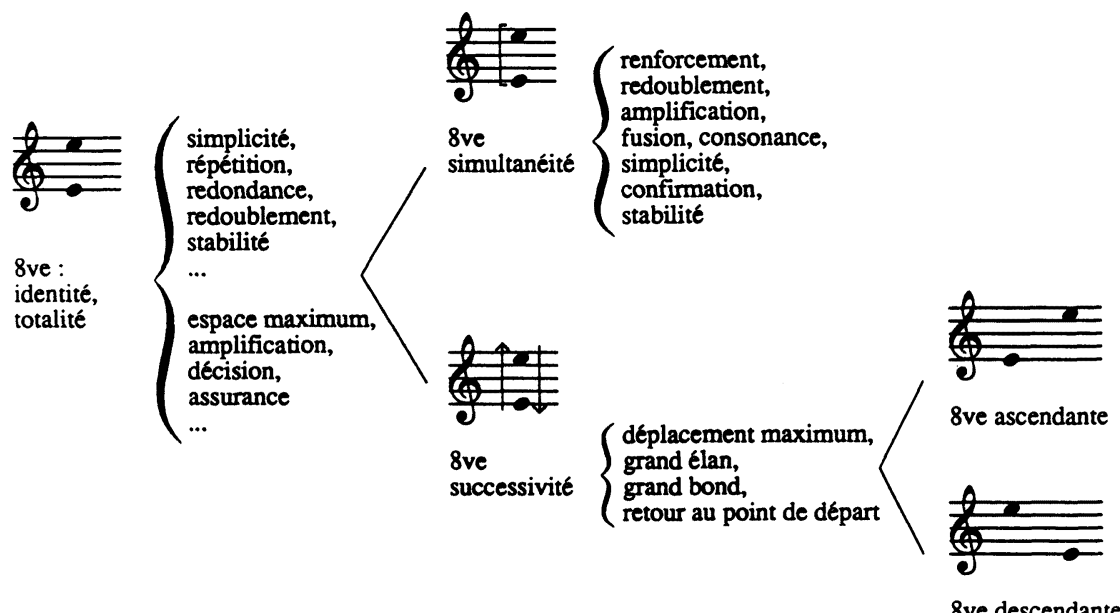

Figure 1. : Premier niveau.

Il importe maintenant de souligner que ce parcours de sens, si ample qu'il soit et, dans cette description peu formalisée, susceptible d'oscillations, doit quand même être considéré comme relativement fermé dans les deux directions, syntaxique et sémantique (une distinction qui, d'ailleurs, n'est pas rigide dans notre modèle). Quant à la syntaxe, l'intervalle tire tout son noyau de sens (identité, totalité) du système fermé de l'échelle à laquelle il appartient ; également fermé est le jeu des articulations simultané/successif, ascendant/descendant. Quant au réseau sémantique, il est ancré uniquement aux deux traits "identité" et 
"totalité", et il se déploie seulement au niveau des codes généraux (logiques, sensoriels, émotionnels) sélectionnés et utilisés d'une manière restrictive.

Ce premier niveau de fonctionnement de notre unité est donc le niveau du système, de la grammaire. ${ }^{2}$ Maintenant, il s'agit de passer à un deuxième niveau, pour voir comment, sur le système, s'enchevêtre le discours, le texte : autrement dit, comment le modèle que nouv avons dessiné fonctionne dans les occurrences concrètes musicales.

B. Au deuxième niveau, celui du discours-texte musical, l'unité grammaticale que nous avons décrite apparait comme un sens virtuel, un potentiel de sens que les contextes musicaux et les circonstances extramusicales (verbales, théâtrales, etc.) peuvent soit neutraliser, soit renforcer et enrichir de nouvelles composantes compatibles avec le modèle.

a) Des exemples typiques de neutralisation (on pourrait dire "d'octave faible") où l'intervalle passe pratiquement inaperçu, ce sont les cas d'octaves simultanées des accords de tutti orchestral, ou bien les cas d'octaves successives dans des dessins mélodiques bâtis avec des intervalles amples et asymétriques :
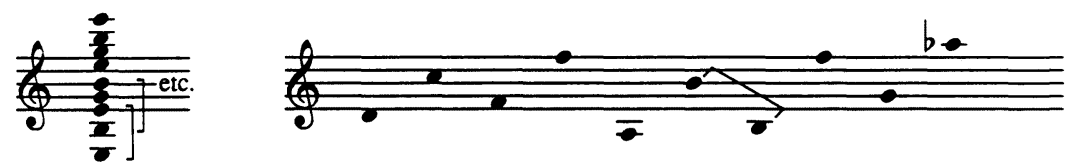

Figure 2.

2 Ici et plus loin, nous faisons référence à la théorie linguistique exposée par Lyons (1968), en particulier en ce qui concerne la "signification lexicale" (1968: 9.2). 
Dans ces cas, les stratégies du discours musical englobent d'une manière évidente et indiscutable l'unité intervallique dans des unités majeures telles que des "accords" ou une "mélodie à grands bonds". Le potentiel de sens de notre unité ici s'exténue, son statut grammatical devient plus restreint ; on serait tenté de ne considérer l'intervalle que comme une simple unité phonologique, c'est-à-dire un élément dépourvu d'un sens à lui, tel qu'un phonème, ou une syllabe, ou un trait d'une séquence programmée (par exemple, la trajectoire d'un bras dans le programme gestuel complexe d'un danseur ou d'un mime). Mais l'enquête précédente nous met en garde : peut-on vraiment, et à quelles conditions, affirmer que le potentiel de sens du premier niveau est rendu ici totalement nul ? Le sens d'identité et de totalité qu'on ressent dans un accord de tutti orchestral ne doit-il pas beaucoup à l'octave ? Donc, au moment où nous constatons pour notre unité grammaticale la tendance à une réduction ou régression phonologique, nous devenons aussi conscients que cette tendance a des modes et des degrés infinis, et qu'une réduction radicale à un degré zéro sera bien difficile à démontrer.

b) Comme exemple d"octave forte", nous donnerons celle du début de Trovatore. Voici une analyse-interprétation de ce passage lyrique qui, dans les contextes musicaux et les circonstances extramusicales de l'occurrence, met en relief les aspects pertinents à un "renforcement" de l'identité syntaxico-sémantique de l'octave (au premier niveau, nous développons ici uniquement l'articulation "descendante", renvoyant, pour le reste, au schéma précédent) : 


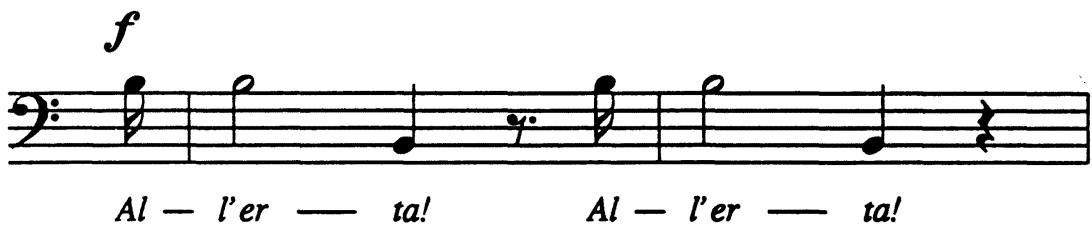

\begin{tabular}{|c|c|c|c|c|}
\hline NIVEAU 1 & \multicolumn{4}{|c|}{ NIVEAU 2} \\
\hline $\begin{array}{l}\text { Articulation } \\
\text { descendante } \\
\text { grande descente, } \\
\text { chute raide, } \\
\text { grand déploierent } \\
\text { d'énergie, } \\
\text { répétition, } \\
\text { retour au point } \\
\text { de départ, } \\
\text { emphase }\end{array}$ & $\begin{array}{l}\text { dominante } \\
\text { anacrouse } \\
\text { forte } \\
\text { solo } \\
\text { phrase } \\
\text { isolée } \\
\text { phrase } \\
\text { répétée }\end{array}$ & $\begin{array}{l}\text { suspense } \\
\text { inpulsion } \\
\text { emphatique, } \\
\text { énergie } \\
\text { évidence } \\
\text { relief } \\
\text { insistance }\end{array}$ & $\begin{array}{l}\begin{array}{l}\text { Circonstan } \\
\text { (libretto) }\end{array} \\
\text { “All'erta!" } \\
\text { Ferrando, } \\
\text { chef des } \\
\text { gardes, } \\
\text { s'adresse } \\
\text { à elles }\end{array}$ & $\begin{array}{l}\text { es } \\
\text { exclama- } \\
\text { tion, } \\
\text { signal, } \\
\text { appel, } \\
\text { cri, } \\
\text { autorité, } \\
\text { ordre, } \\
\text { décision } \\
\text { pérerptoire }\end{array}$ \\
\hline
\end{tabular}

Figure 3.

Le sens global de cet épisode lyrique (cri rituel, signal solennel, appel militaire) peut être envisagé comme une expansion (intensification et extension) du sens de base de l'intervalle d'octave ; d'après le schéma 
ci-dessus, il ne serait pas difficile de montrer plus en détail la construction progressive, par traits et codes, de ce sens global à partir du sens grammatical de l'octave.

De cet exemple, on peut inférer une règle générale : le renforcement consiste à ramener autant que possible l'unité textuelle ou discursive à l'unité grammaticale ou systémique ; or, cela peut être fait soit sur le plan syntaxique, par isomorphisme, soit sur le plan sémantique, par isotopie. Dans l'exemple de Trovatore, l'intervalle d'octave constitue par lui seul l'cénoncé" musical quant à la dimension diastématique : l'unité grammaticale coïncide ainsi avec l'unité textuelle, est isomorphe à celle-ci, s'impose par elle-même à l'attention de l'auditeur ; bref, l'intervalle sera d'autant plus un énoncé bien individualisé qu'il sera isolé - comme il est ici - ou dans une position privilégiée, par exemple, au début ou à la fin d'une phrase ou d'un morceau. Quant aux aspects musicaux et extramusicaux concourant à cette individuation dans l'exemple choisi, il est aisé de remarquer : l'isomorphisme entre la "grande chute de voix" de l'exclamation-cri dans l'expression orale, et la "grande chute" de l'octave descendante ; les isotopies "répétition", "emphase", "énergie", etc., résultant de la convergence des contextes musicaux et des circonstances théâtrales.

Pas besoin d'une compétence verdienne particulière pour se rendre compte qu'on est en face d'un exemple typique. Combien de cris et d'exclamations dans Verdi sont bâtis sur une octave descendante (rien que dans Trovatore, il y en a plusieurs dizaines) ; et pas seulement dans Verdi, mais dans toute l'histoire de l'opéra, et en général, dans l'histoire du chant. C'est pourquoi ce fonctionnement syntaxico-sémantique de l'intervalle d'octave descendante, qui se donne à l'évidence comme une stylisation de la chute de voix typique de l'exclamation orale, nous autorise à parler d'une "octave exclamative". 
c) Un signifiant musical donné - l'intervalle d'octave descendante est ainsi corrélé à un signifié - l'exclamation - d'une manière constante. Nous sommes donc en présence d'un code (musicalparalinguistique) et d'une unité de signification ayant un statut quasi-lexical, une sorte de "parole musicale".

Et puisque, comme nous l'avons vu, cette identité lexicale présuppose, englobe, renforce et enrichit une identité grammaticale, nous pouvons résumer le modèle théorique de l'intervalle d'octave dans le schéma d'ensemble suivant :

PREMIER

NIVEAU:

système

DEUXIÈME

NIVEAU:

discours

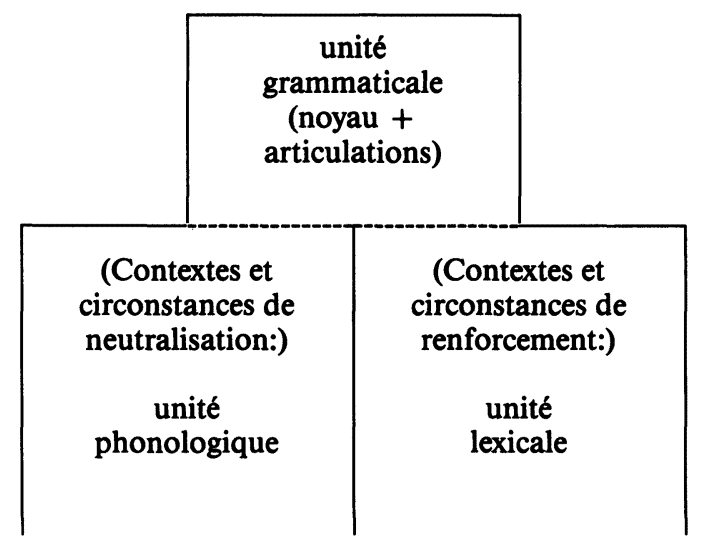

Figure 4. 
d) Une fois mis en marche, le processus de reconnaissance de certaines identités lexicales de l'octave ne s'arrête pas au cas décrit ci-dessus. Il est évident, en effet, que l'octave n'est pas seulement "exclamative". On peut donc prévoir de parvenir à l'individuation d'une série de significations, c'est-à-dire à des "acceptions" constantes et prédominantes de l'octave qu'on peut ranger comme dans un "dictionnaire". Par exemple, les suivantes :

- octave "gestuelle" : simultanée, très évidente dans les "stretti" symphoniques ou lyriques où les instruments et les voix, parvenus à un climax dramatique, resserrent les rangs et unifient les rôles, et où le jeu musical se condense dans une action à l'unisson. Ou alors, octave successive, dans ces genres d'attaques qui fonctionnent comme des décharges soudaines d'énergie (par exemple, le Scherzo dans la Neuvième Symphonie de Beethoven), ou ceux qui miment des mouvements rituels de grande solennité (par exemple, l'Adagio du Concerto en La mineur, de Vivaldi, transcrit pour orgue par Bach) ;

- octave "figurative" : renforcement de la mélodie vocale au moyen d'instruments, ou l'usage habituel des premiers et seconds violons qui jouent à l'octave dans l'orchestre classique - une figure sur un fond ;

- octave "de virtuosite" : l'octave étant le "saut" le plus grand dans le système, elle fonctionne souvent, dans des passages instrumentaux ou vocaux, comme un effet de technique extraordinaire, comme un effet de bravoure, comme quelque chose de spectaculaire - c'est un saut athlétique sur l'échelle.

e) Nous avions remarqué que le sens "grammatical" de l'octave se construit uniquement au niveau des codes généraux (logico-sensorimoteurs-émotifs); maintenant, nous pouvons remarquer que la "régression phonologique" et, plus encore, le renforcement lexical s'opère 
à l'aide de codes de niveaux plus complexes : pratiques sociales, techniques et styles musicaux. Par exemple, l'identité lexicale "octave exclamative" se détermine de manière essentielle par un code paralinguistique, donc une pratique sociale. L'identité "gestuelle" utilise des codes qui réfèrent à des conduites plus ou moins socialis\& eac.es. Une acception "figurative" de l'octave passe à travers des structures perceptives où se condensent aussi des conventions compositionnelles. Finalement, ce sont des pratiques et techniques spécifiquement musicales qui codifient davantage l'acception de "virtuosité" de l'octave. Et il est presque oiseux d'ajouter que cette production de sens implique une compétence tout aussi bien au niveau des styles musicaux.

\section{Perspectives et problèmes}

\section{A. Vérification et extension}

a) A la lecture ou à l'écoute de pièces de musique, il arrive souvent de constater que le seuil d'individuation de l'intervalle d'octave est oscillant (ce qui vaut, d'ailleurs, mais avec des modalités différentes, pour tous les intervalles) : et cela, non seulement pour la perception d'une "identité lexicale", mais déjà pour la simple perception "syntaxique". Par exemple : un glissando d'octave est-il encore perçu comme un "intervalle" d'octave ? et une coulée très rapide de double-croches ? et un passage moins rapide sur l'échelle, telle l'attaque du quatrième mouvement de la Première Symphonie de Beethoven ? et le parcours-ambitus (d'octave) d'une phrase mélodique comme "Celeste Aïda" ?

Ces données de l'expérience nous amènent à élargir notre théorie. Nous dirons que, dans la mesure où il perd en "intensité" ce qu'il gagne en "extension", l'intervalle d'octave cesse de fonctionner comme unité grammaticale pour devenir graduellement une stratégie textuelle ou un programme narratif. Notre unité "linguistique" a donc, pour ainsi dire, une articulation multiple qui est en même temps relativement souple ; 
elle peut se représenter comme une échelle quasi-continue où les contextes et les circonstances concourent à rendre pertinent chaque fois tel ou tel niveau :

$\begin{array}{lcc}\begin{array}{l}\text { articulation } \\ \text { minimale }\end{array} & & \begin{array}{c}\text { articulation } \\ \text { maximale }\end{array} \\ & \text { phonologique grammatical lexical narrative } & \end{array}$

Figure 5.

Il reste à ajouter que les octaves "diluées" ou "remplies" du niveau narratif gardent, elles aussi, leur sens grammatical de base ; en d'autres mots, les transformations sémantiques sont conformes à celles de la syntaxe.

b) Peut-on appliquer ce modèle à d'autres intervalles ? En principe, rien ne devrait l'empêcher. Chaque intervalle montre, d'une manière qui s'impose plus ou moins, quelques émergences sémantiques sur lesquelles peut s'appuyer une recherche telle que nous l'avons menée pour l'octave. Tout comme pour l'octave, le sens de chaque intervalle va ressortir du système de l'échelle, puis des articulations spatiotemporelles, puis des contextes et des circonstances.

Dans la pratique, bien entendu, la réalisation de la recherce ainsi que les contenus du modèle se présentent, pour chaque intervalle, à un degré différent de complexité et d'exigence de travail. On le comprend bien si l'on réfléchit aux différentes fonctions de chaque intervalle dans les régimes modaux, les conduites mélodiques et harmoniques, le système tonal, bref, dans toutes les stratifications de sens englobées dans l'échelle et les intervalles. Nous allons en voir rapidement un exemple pour l'intervalle de quinte.

On relèvera quelques occurrences significatives, avec relief syntaxique et densité sémantique, pour amorcer la recherche sur l'intervalle de 
quinte : i) les attaques ascendantes typiques des intonations récitatives archaïques (épitaphe de Seikilos, introït grégorien Puer natus est, ballade anglaise Henry Martin dans une version très répandue, etc.), qu'on retrouve dans bien des contextes classiques et dans les musiques de film évoquant les "grandes distances" dans le temps et l'espace, et qui pourraient trouver leur exemple typique dans le solo de cor anglais de Tristan ; ii) les quintes "vides" simultanées fonctionnant en "bourdon" et évoquant dans bien des cas le pastoral (Beethoven, Sixième Symphonie). Cet intervalle étant bien codé dans les régimes modaux et le système tonal-harmonique, son noyau de sens sur l'échelle n'est pas difficile à définir (si on laisse de côté certaines ambiguïtés qui l'apparentent à la quarte). Voici, en synthèse, ce que pourrait être son modèle au niveau 1 :

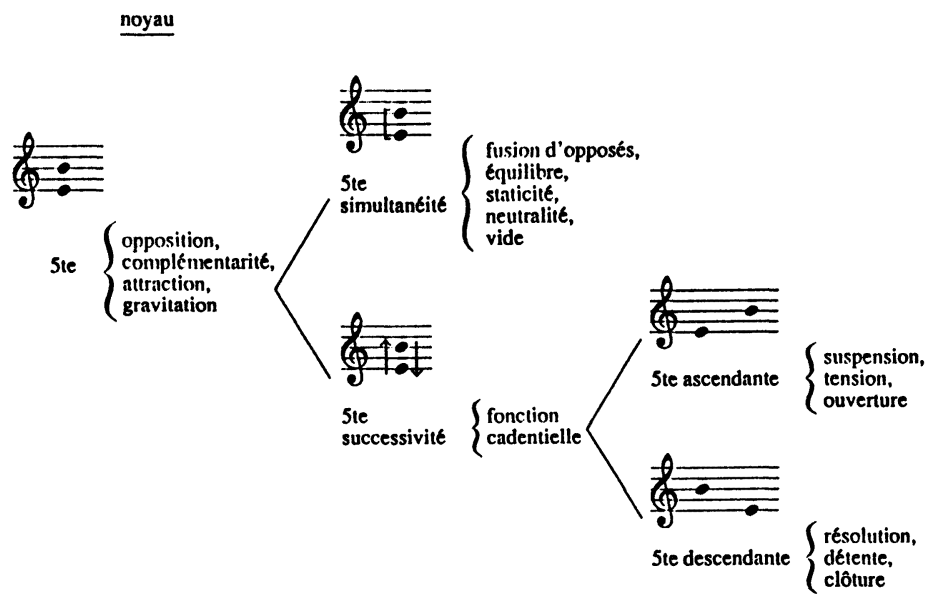

Figure 6. 
Quant au niveau 2, on peut discerner facilement les "quintes d'intonation" et les "quintes de bourdon"; mais on serait tenté d'y ajouter tout de suite les "quintes de cor"...

Une situation très différente est celle de l'intervalle de seconde. Son faible codage dans les systèmes dominants (tonal et harmonique) oblige à préciser le sens de cet intervalle dans des domaines et réseaux peu explorés par la théorie musicale : l'opposition des degrés conjoints/disjoints ; le rôle non essentiel ou dérangeant de la seconde dans le code accordal qui considère comme unité élémentaire la tierce; le chromatisme comme articulation subliminaire, donc également non essentiel et bruyant, du régime scalaire normal, c'est-àdire diatonique ; le code des “ornements" dont le plan du contenu n'est pas seulement l'esthétique, mais aussi l'expressif, le descriptif, le paralinguistique, etc. ; les modes, gammes et formules mélodiques "élémentaires" au sens de Brăiloiu ou Szabolcsi. ${ }^{3}$

\section{B. Théorie et analyse}

On peut résumer notre théorie selon les points suivants :

1. l'intervalle est une unité syntaxique douée de signifiance (senssignification) ;

2. sa signifiance radicale lui vient de sa fonction à l'intérieur de l'échelle, et précisément du fait qu'il est une relation et une distance-ambitus entre deux notes données de l'échelle ;

3. dans les occurrences concrètes, cette signifiance radicale peut être ou bien neutralisée partiellement, ou bien renforcée et amplifiée par les contextes et les circonstances ;

3 Cf. "L'intervallo di seconda", in Stefani (1982: 19-84). 
4. le fonctionnement "linguistique" d'un intervalle se réalise à des niveaux d'articulation différents et mobiles (phonologique, grammatical, lexical, narratif).

Or, dans quelle relation se pose notre théorie avec les théories courantes sur les intervalles? Passons en revue quelques cas typiques.

a) Théorie acoustique. Dans notre théorie, tout comme dans les grammaires musicales courantes, l'intervalle est un élément spécifiquement musical, puisqu'il prend son sens par rapport au systèmelangage musical qu'est l'échelle. Comme pour tout fait linguistique, ce qui est pertinent ici, ce n'est que la forme ou identité phonologique, constituée par les relations fonctionnelles entre les notes de l'échelle ; par contre, est indifférente la substance ou identité phonétique, représentéee par les "hauteurs absolues" des sons, leurs mesures quantitatives et leurs relations avec les causes physiques qui les produisent. ${ }^{4}$ Or, c'est justement cette substance phonétique qui fait l'objet de la théorie acoustique.

A partir de cette diversité et indépendance essentielle des deux approches, on peut prendre en charge quelques problèmes de frontière. Le plus commun nous semble l'évidence perceptive incontestable de certains intervalles, en particulier l'octave et la quinte, qui auraient un certain caractère de "naturalité" et de fondement par rapport aux systèmes musicaux. Or, la linguistique, elle aussi, s'est trouvée en face d'un problème parallèle, et la solution qu'elle a trouvée nous semble satisfaisante aussi pour notre cas. Pour des raisons physiques et physiologiques, certains sons s'avèrent plus aisés à produire ou à percevoir que d'autres ; c'est pourquoi on les rencontre plus fréquemment et, parce qu'ils sont aisément reconnaissables, il sont plus aptes à assurer des fonctions importantes dans un système donné. Il est donc vrai que leur choix a été plus motivé, moins arbitraire que celui d'autres

4 Pour tout ce paragraphe, cf. Lyons (1968:2.2, "Substance et forme"). 
éléments ; mais ceci ne doit pas faire oublier que, une fois qu'ils sont dans le système, ce qui compte est la fonction que le système leur assigne ; si bien que la motivation peut très bien disparaître, et, de fait, disparaît souvent.

Ainsi, il n'y a pas de raison pour nier que la substance phonétique ait été historiquement motivante pour la forme phonologique de l'intervalle d'octave, et qu'une telle motivation demeure active en ce sens que, dans cet intervalle (si on le joue "juste"), nous pouvons entendre "le même son à hauteurs différentes", même indépendamment de la pertinence scalaire (modale-tonale). Mais c'est une erreur théorique que de confondre cette similarité perceptive avec l'identité fonctionnelle ; et l'erreur devient plus grave lorsqu'on mêle les contructions spéculatives sur la substance phonétique (théories des harmoniques et des proportions) avec les systèmes phonologiques (modes, gammes, échelles, etc.) sur lesquels se sont réellement fondés et se fondent l'expérience et le langage de la musique dans les sociétés occidentales. Une telle confusion empêche certainement l'explication du fonctionnement culturel complexe d'un intervalle tel que la seconde ; elle ne devrait pas être tolérée davantage dans une musicologie alertée par la linguistique et la sémiotique.

b) Grammaire. En gros, on peut dire que la grammaire musicale traditionnelle vise uniquement la face syntaxique des structures musicales sans rapport avec la sémantique, et cela, parce qu'elle ne tient pas compte d'une pragmatique. Autrement dit, elle est une théorie formelle organisant des éléments et des règles, mais elle n'est pas une grammaire linguistique puisqu'elle n'explicite pas le fonctionnement sémiotique de ces éléments et règles. If faut encore ajouter que, puisqu'elle ne considère pas la signifiance musicale dans sa complexité de pertinences, la théorie traditionnelle n'arrive pas à définir et coordonner les différents niveaux d'organisation même syntaxique des éléments eux-mêmes. ${ }^{5}$ En

5 Cf. ma critique de la grammaire de Karolyi in Stefani (1982: 80-84). 
fait, une théorie satisfaisante des intervalles devrait coordonner de façon organique : les pertinences logico-sensori-motrices (vecteurs ascendant/descendant, procédés conjoint/disjoint, propriétés métriques, de grandeur et de périodicité, etc.) ; les codes oraux-modaux (par exemple, les intonations récitatives, organisées autour des fonctions de initium, ténor, cadence) ${ }^{6}$; le système tonal qui se greffe sur ces codes et les règles harmoniques, soit dans leur dérivation du système tonal soit dans ce qu'elles ont en propre ; les schèmes combinatoires, plus ou moins arbitraires ou motivés, inventés par les musiciens de l'avant-garde historique. ${ }^{7}$

c) Sémantique. La dimension sémantique est inéluctable, si l'on veut expliquer la syntaxe de bien des oeuvres musicales, en premier lieu dans le domaine de l'opéra. En fait, c'est à partir d'un travail interprétatif dans ce domaine que Deryck Cooke a développé sa sémantique des intervalles (1959 : 40-90). Nous avons discuté ailleurs (Stefani 1982) ses descriptions d'un intervalle particulier, la seconde ; ici, nous voudrions résumer ses positions générales.

1. Cooke introduit dans son discours les données de la théorie acoustique des harmoniques directement en tant que contenus sémantiques des intervalles ; en cela, il va être concerné par la critique que nous avons adressée à la théorie acoustique.

2. Parmi les codes syntaxiques, il privilégie de manière presque exclusive le code tonal : sa théorie présente donc les limites indiquées ci-dessus pour la grammaire courante.

6 Dans ce domaine, on valorise encore peu les contributions de chercheurs comme Smits van Waesberghe, Szabolcsi, Brăiloiu, qu'il faudrait intégrer dans une théorie générale des intervalles.

7 Bartók est certainement un compositeur exemplaire à cet égard, quant à l'invention et la synthèse de tous ces niveaux de sens. Sur certains aspects de sa sagesse combinatoire, cf. Lendval (1982). 
3. Pour Cooke, les contenus sémantiques des intervalles se réduisent à l'émotif ; il lui manque une optique suffisamment ample de la signifiance en musique (qui fait l'objet de notre modèle de compétence musicale).

4. Il manque également à Cooke une notion de l'articulation linguistique, voire une perspective sur le fonctionnement des intervalles à différents niveaux d'articulation. Cela entraîne aussi une grave limite de méthode : les attributions de signifiance aux intervalles, dans les différentes occurrences, sont faites d'une façon globale, sans discrimination ; c'est pourquoi elles sont plus ou moins convaincantes, et parfois pas du tout, l'auteur ne se fondant sur aucune démonstration sinon sur son intuition personnelle, laquelle, d'ailleurs, n'est pas tout à fait banale.

d) Analyse. Le musicologue hongrois Peter P. Varnai a consacré un essai (1969) à ce qu'il appelle l'“unisson" (mais qui, en réalité, est plutôt l'intervalle d'octave dans cette identité lexicale que nous avons appelée "gestuelle") dans quelques oeuvres de Mozart.

Son intuition de départ - qu'ensuite il s'efforcera simplement de réaffirmer par une accumulation d'exemples - est la suivante : "L'unisson apparaît ici le moyen le plus important pour exprimer un monde de sentiments négatifs [...]. Pour Mozart, tout comme pour son époque et même 'avant' et 'après' lui, l'unisson de la voix et de l'orchestre, parfois simplement l'unisson de l'orchestre, constitue le moyen musical expressif par excellence en vue de révéler le monde des concepts négatifs" (1969 : 423) $)^{8}$ comme le mal, le désagréable, le mépris, les enfers, etc.

8 "L'unisono si presenta qui come il più importante dei mezzi per esprimere un mondo sentimentale negativo [...]. Sia per Mozart, sia per la sua epoca ed anche 'prima' e 'dopo' di lui, l'unisono della voce e dell'orchestra, a volte soltanto l'unisono dell'orchestra, costituisce il mezzo musicale espressivo per eccellenza allo scopo di rivelare il mondo dei concetti negativi." 
Il est évidemment trop facile de contester cette affirmation, soit à l'aide d'exemples contraires contemporains de Mozart, soit parce que c'est presque un truisme de remarquer qu'un élément stable, clair, rassurant, institutionnel comme l'octave ne peut être corrélé, dans la société de l'ancien régime surtout, à aucun pôle négatif. En revanche, il est intéressant de déceler les raisons par lesquelles un analyste peut perdre le nord de cette manière.

Varnai part du présupposé - implicite, peut-être inconscient - que l'intervalle n'a pas en soi, et en tant qu'unité grammaticale, de signifiance ; la signifiance surgirait seulement en vertu de conventions rhétoriques ultérieures : "La mission spécifique de la musique était justement la représentation des expressions sentimentales : les passions. Dans les nombreux traités publiés à l'époque sur l'esthétique, un système tout entier en est élaboré, et les passions fournissent au compositeur un véritable vocabulaire. A notre avis, l'unisson [c'est-à-dire l'octave] lui aussi, en tant que moyen expressif de sentiments négatifs, appartient aux différentes 'manières' ainsi élaborées et cofidiées" (1969: 424).9

On voit bien que nous sommes conduits maintenant à critiquer non seulement Varnai, mais l'Affektenlehre et la Figurenlehre, du moins dans une certaine interprétation moderne. Notre hypothèse veut, en fait, que les répertoires baroques des figures rhétoriques et des affects (répertoires, et non pas systèmes, comme le pense Varnai) étaient conçus par les contemporains comme un "niveau 2" de signifiance qui en présuppose un premier, grammatical et systémique. Par là, toute recherche sur les figures ou la figuralité chez Bach ou Schütz devraient rester sub judice en attendant qu'on éclaircisse le fonctionnement

9 "La specifica missione della musica era appunto la rappresentazione delle espressioni sentimentali : gli affetti. Nei numerosi trattati pubblicati allora sull' estetica viene elaborato un intero sistema, ed essi forniscono al compositore un vero e proprio vocabulario. Secondo noi, anche l'unisono quale mezzo espressivo di sentimenti negativi appartiene alle varie 'maniere' cosi elaborate e codificate." 
grammatical des intervalles auquel les figures se relient et sont ancrées. Quel qu'ait pu être le changement des contextes et des circonstances, on peut prévoir qu'un même réseau d'identités lexicales sous-tende, en partie au moins, la figuralité baroque et celle, romantique, d'un Schumann et d'un Berlioz, à part, bien entendu, celle d'un Verdi.

D'autre part, le code baroque des figures ne semble offrir à Varnai aucun support dans son cas spécifique, contrairement à ce qu'il déclare. D'où vient donc la signifiance de l'intervalle d'octave-unisson ? Il n'y a pas de choix : immédiatement des circonstances, c'est-à-dire du libretto. L'expression musicale, en soi vide et neutre, se remplit du contenu de l'expression verbale :

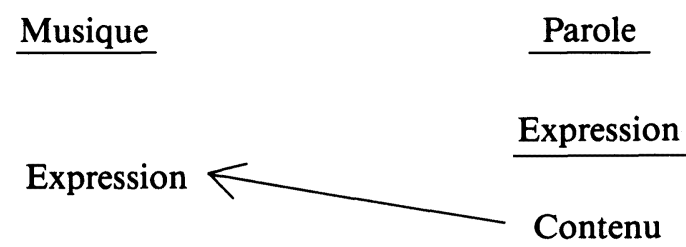

Figure 7.

Or, un tel remplissage est trompeur puisqu'il réduit à trois termes un rapport sémiotique (rapport musique-parole) qui, en réalité, en a quatre. 10

10 Cf. N. Ruwet, "Fonction de la parole dans la musique vocale" (1961) in Ruwet (1972 : 41-69) ; G. Stefani, "Poesia e musica: i codici della lirica" in Stefani (1976 : 178-199) ; R. Dalmonte, "il Nachtlied di GoetheSchumann : proposte e verifica di un metodo d'analisi", (Dalmonte 1980). 
Mise à part sa position sémiotique fallacieuse, le travail de Varnai pose le problème suivant : est-il possible d'interpréter un texte au niveau du style en faisant abstraction des niveaux de système ? est-il possible d'analyser un discours en faisant abstraction d'une grammaire ? est-il possible d'utiliser un terme dans un texte en ignorant ses acceptions de dictionnaire ? Notre modèle n'exclut pas cette possibilité théorique, mais il en précise l'improbabilité et prévoit les risques de distorsions ou, comme c'est le cas ici, d'aberrations.

Si l'analyse de Varnai portait sur les niveaux de style d'auteur et d'époque, celle de Gazzaniga (1979) sur l'intervalle de sixte se situe au niveau de l'oeuvre, recherchant le fonctionnement sémantique de cet intervalle dans Lucia di Lammermoor. Gazzaniga fait nettement abstraction des niveaux de sens qui précèdent, ceux du style et de la grammaire. Après avoir déclaré que la sixte "est un intervalle des plus communs et des plus utilisés, n'ayant rien de bruyant, d'exceptionnel" (1979:631)11, il s'efforce de démontrer que Donizetti, dans les dernières scènes de Lucia, prend cet intervalle - dans sa forme ascendante - "comme une matière première absolument amorphe, uniquement réglée par les lois tonales" (il ne précise pas lesquelles), qui "évolue, malgré son apparence tranquille, dans une grande variété de vibrations" (1979: 632)12 se constituant en "moyen pour signifier la résignation désolée à la tragédie" (1979: 625)13 pour exprimer (ou susciter) "soit l'invective exaltée, soit la résignation extrême, désespérée" (1979 : 628). ${ }^{14}$ A un

11 "E un intervallo dei più comuni e dei più usati ; e non ha nulla di improvvisamente eccezionale, di clamoroso."

12 "Come una materia prima assolutamente amorfa, solo regolata dalle leggi tonali ... evolvendosi, nonostante la tranquilla apparenza, in una variegatissima sorta di vibrazioni."

13 "Mezzo per significare la desolata rassegnazione alla tragedia."

14 "Sia l'esaltata invettiva sia l'estrema, disperata rassegnazione." 
moment donné, par décision de l'analyste, l'intervalle se révèle comme une véritable "catégorie lexicale", où "l'émotion provoquée par (ou contenue dans) ce signe revient avec son identité propre, sa connotation, sa destinée" (1979:629)15, bref, avec son code défini et définitif, bien que valable uniquement pour ces contextes et circonstances.

Réfléchissons : si un intervalle n'a pas de noyau sémantique à lui, il deviendra logiquement le lieu neutre de manifestation d'un sens qui se produit sans son concours sémantique ; nous l'avions déjà remarqué à propos de Varnai. Mais jusqu'où va s'étendre ce principe de neutralité des signifiants musicaux ? et où donc va se produire, alors, le sens musical ? On est dans une impasse théorique : ou bien Gazzaniga se borne à décrire le contenu théâtral dans son ensemble (résignation désolée à la tragédie, etc.), sans aucune référence analytique à l'expression musicale, ou bien, s'il fait ces références, il devra justifier cette pertinence d'une manière bien plus solide et détaillée.

Avec des bases semblables, une exégèse ne rendra compte ni du sens du texte musical ni du sens des éléments considérés pertinents. Pourquoi l'intervalle de sixte devrait-il concourir positivement à construire cette "résignation désolée" ? Ne serait-ce peut-être pas d'autres facteurs qui déterminent ce sens, éventuellement malgré la présence de la sixte ? Gazzaniga a fait l'erreur de méthode de ne pas partir d'une émergence significative de l'intervalle qu'il voulait étudier ; s'il voulait reprendre son étude, on pourrait lui suggérer des exemples tels que l'attaque de "Libiamo nei lieti calici" dans Traviata, ou de "Esultate !" dans Otello, de Verdi. Il pourrait ainsi découvrir que, chez Verdi, la sixte majeure ascendante est un intervalle de l'euphorie, de l'optimisme, et une enquête sur le niveau 1 lui en fournirait les fondements.

15 "L'emozione provocata da (o contenuta in) quel segno, ritorna colla propria identità, con la propria connotazione, col suo proprio destino." 


\section{RÉFÉRENCES}

COOKE, D.

1959: The Language of Music. London : Oxford University Press.

DALMONTE, R.

1980: "Il Nachtlied di Goethe-Schumann : proposte e verifica di un metodo d'analisi", Ricerche Musicali, IV, 81-111.

GAZZANIGA, A.

1979: "Un intervallo nelle ultime scene di 'Lucia", Nuova Rivista Musicale Italiana, 1979/3, 620-633.

LENDVAL, E.

1982: "La Sezione Aurea nelle strutture musicali bartokiane", Nuova Rivista Musicale Italiana, 1982/2, 157-181, 1982/3, 304-399.

LYONS J.

1968: Introduction to Theoretical Linguistics. London : Cambridge University Press.

RUWET, N.

1972: Langage, musique, poésie. Paris : Seuil, 1972.

STEFANI, G.

1976: Introduzione alla semiotica della musica. Palermo : Sellerio.

1981: "Eine Theorie der musikalischen Kompetenz", in H.H. Henze (ed.), Die Zeichen. Neue Aspekte der musikalischen Aesthetik, II, Frankfurt : Fischer, 157-178.

1982: La competenza musicale. Bologna : Cooperativa Libraria Universitaria Editrice.

VARNAI, P.P.

1969: "La funzione dell'unisono nella drammaturgia di Mozart", Nuova Rivista Musicale Italiana, 1969/3, 422-438. 